nation of them is an invariant of $G$. We can find* a product of such combinations which has integral coefficients and vanishes at any assigned point, not a special point. Thus the invariant is the product of one or more such products.

For $d$ odd, a non-special point is one of $p d$ conjugates under $G$. We now use the absolute invariants $y^{p d}, \lambda^{d}$.

ThEOREM. As a fundamental system of invariants of a group of transformations (1), we may take $y$ and $\lambda$.

In particular, this theorem yields the seminvariant leaders of invariants of two pairs of cogredient variables.

University of Chicago,

February, 1913.

\title{
ON SOME SYSTEMS OF COLLINEATION GROUPS.
}

\author{
BY DR. HOWARD H. MITCHELL.
}

(Read before the American Mathematical Society, April 26, 1913.)

$\S 1$.

Some systems of collineation groups which arise in connection with the theory of elliptic functions have been investigated by Klein $\nmid$ and Hurwitz $\ddagger$. One of them is a system in $n$ variables each group of which contains an invariant subgroup of order $n^{2}$. For $n$ a prime the quotient group with respect to this invariant subgroup is $(1,1)$ isomorphic with the modular group on two indices of order $n\left(n^{2}-1\right)$. The group in three variables is the Hessian group of order 216.

For $n$ odd there is also an invariant subgroup of order $2 n^{2}$, and there exist two other groups in $(n-1) / 2$ and $(n+1) / 2$ variables each of which is isomorphic with the quotient group with respect to this subgroup. Thus for $n=5$ there is both a binary and a ternary $G_{60}$ and for $n=7$ both a ternary and a quaternary $G_{168}$.

Similar systems of groups in $n^{2},\left(n^{2}-1\right) / 2$, and $\left(n^{2}+1\right) / 2$ variables which arise in the theory of hyperelliptic functions

* Dickson, Trans. Amer. Math. Soc., vol. 12 (1911), p. 4.

$\dagger$ Math. Annalen, vol. 15 (1879), p. 275; also Klein-Fricke, Modulfunktionen (2) 5 .

$\ddagger$ Math. Annalen, vol. 27 (1885), p. 198. 
have been investigated by Witting* and Burkhardt. $\dagger$ The group in $n^{2}$ variables for $n$ a prime is isomorphic with the abelian linear group on four indices with modulus $n$.

For $n$ odd there is an invariant subgroup of order 2 , and the groups in $\left(n^{2}-1\right) / 2$ and $\left(n^{2}+1\right) / 2$ variables are $(1,1)$ isomorphic with the quotient group with respect to this subgroup. Thus for $n=3$ there is both a quaternary and a quinary group of order 25920 .

The groups in $n$ and $n^{2}$ variables, when $n$ is a prime $p$, are closely connected with a group in $p^{m}$ variables investigated by Jordan $\ddagger$ and Dickson. $\S$ As a collineation group it contains an invariant subgroup of order $p^{2 m}$ and the quotient group is $(1,1)$ isomorphic with the abelian linear group on $2 m$ indices and coefficients reduced modulo $p$. For $p$ odd there is an invariant subgroup of order $2 p^{2 m}$ and there exist two other groups in $\left(p^{m}-1\right) / 2$ and $\left(p^{m}+1\right) / 2$ variables which are isomorphic with the quotient group with respect to this subgroup. These two groups do not seem to have been noticed before for $m>2$.

All three of these groups are shown by the author to be "transitive" and "primitive" in the variables. These terms in relation to collineation groups seem to have been employed first by Maschke and Blichfeldt.

\section{$\S 2$.}

Jordan and Dickson consider $p^{m}$ variables which are distinguished by $m$ subscripts, $\xi_{1}, \xi_{2}, \cdots, \xi_{m}$, each of which is an integer reduced modulo $p$. This may readily be translated into ordinary numbering if we consider that $\xi_{1}, \xi_{2}, \ldots, \xi_{m}$ represents $\xi_{1}+\xi_{2} p+\cdots+\xi_{m} p^{m-1}$, where the smallest positive (or 0 ) residue is taken.

The group in these variables which they consider is generated by the following operators:

$$
\begin{aligned}
A_{i}: X_{\xi_{1} \cdots \xi_{m}}{ }^{\prime} & =\theta^{\xi_{i}} X_{\xi_{1} \cdots \xi_{m}} ; \\
B_{i}: X_{\xi_{1} \cdots \xi_{\xi} \cdots \xi_{m}} & =X_{\xi_{1} \cdots \xi_{i}+1 \cdots \xi_{m}} ; \\
L_{i}: X_{\xi_{1} \cdots \xi_{m}} & =\theta^{\frac{1}{\xi_{i}\left(\xi_{i}+p\right)} X_{\xi_{1} \cdots \xi_{m}}} ;
\end{aligned}
$$

* Math. Annalen, vol. 29 (1886), p. 157; Diss. (Göttingen, 1887).

† Math. Annalen, vol. 38 (1891), p. 163.

$\ddagger$ Traité des Substitutions, pp. 420-450.

\& Trans. Amer. Math. Soc., vol. 1, pp. 30-38. 


$$
\begin{aligned}
& M_{i}: X_{\xi_{1} \cdots \xi_{i} \cdots \xi_{m}}=\frac{(-1)^{r / 2}}{\sqrt{p}} \sum_{\mu=0}^{p-1} \theta^{\mu \xi_{i}} X_{\xi_{1} \cdots \mu_{\mu} \cdots \xi_{m}} ; \\
& N_{i j}: X_{\xi_{i} \cdots \xi_{m}}{ }^{\prime}=\theta^{\xi_{i \xi_{j}}} X_{\xi_{1} \cdots \xi_{m}} ;
\end{aligned}
$$

where $i, j=1,2, \cdots, m ; j \neq i ; \theta^{p}=1, \theta \neq 1$; and $r$ is so chosen that the determinant of $M_{i}$ is unity. If $p=2$ the exponent of $\theta$ in $L_{i}$ is not reduced modulo 2. The multiplier is then $-\sqrt{-1}$ if $\xi_{i} \equiv 1$, and +1 if $\xi_{i} \equiv 0, \bmod 2$. In this case $L_{i}$ is of period 4 , and for $p$ odd it is of period $p$.

The group leaves invariant the subgroup of order* $p^{2 m}$ generated by the $2 m$ operators, $A_{i}, B_{i}$. If any operator of this subgroup be expressed in the form

$$
\prod_{i=1}^{m} A_{i}^{x_{i}} B_{i}^{y_{i}},
$$

it is shown by Jordan that the substitutions on the $2 m$ indices $x_{i}, y_{i}$ which are effected when $L_{i}, M_{i}, N_{i j}$ are used to transform this general operator, generate the abelian linear group $\dagger$ on those $2 m$ indices. The operators $L_{i}$ as written here are equal to the $L_{i}$ of Jordan by a power of $A_{i}$, but since $A_{i}$ leaves all the indices unaltered, the resulting substitution is the same.

If $p>2$, the group on these $2 m$ indices contains an invariant substitution which changes the sign of each of them. One of the collineations which effects this substitution on the $2 m$ indices is

$$
R: \quad X_{\xi_{1} \cdots \xi_{m}}=(-1)^{\frac{1}{\left(\left(p^{m}-1\right)\right.}} X_{-\xi_{1} \cdots-\xi_{m}} .
$$

This transformation is commutative with $L_{i}, M_{i}$, and $N_{i j}$ and is transformed into $p^{2 m}$ conjugate operators by the invariant subgroup. Hence the group commutative with $R$ is $(1,1)$ isomorphic with the abelian linear group.

It will be observed that $R$ multiplies by \pm 1 each of the $\left(p^{m}+1\right) / 2$ linear expressions

$$
X_{0 \cdots 0}, \quad X_{\xi_{1} \cdots \xi_{m}}+X_{-\xi_{1} \cdots-\xi_{m}},
$$

and multiplies by $\mp 1$ each of the $\left(p^{m}-1\right) / 2$ expressions

$$
X_{\xi_{1} \cdots \xi_{m}}-X_{-\xi_{1} \cdots-\xi_{m}},
$$

* As a linear group this subgroup is of order $p^{2 m+1}$ if $p$ is odd and $2^{2 m+2}$ if $p=2$; in the latter case an additional generator being necessary.

† L. c.; also Dickson, Linear Groups, p. 92. 
where not every $\xi_{i}$ is 0 . Each of the transformations $L_{i}$, $M_{i}, N_{i j}$ therefore transforms any one of these expressions into a linear combination of the others in the same set. Hence there exist collineation groups in both $\left(p^{m}+1\right) / 2$ and $\left(p^{m}-1\right) / 2$ variables which are isomorphic with the abelian linear group. Since the quotient group of that group with respect to its invariant operator of period 2 is simple except in the case $p^{m}=3^{1}$, it follows that the group in each set of variables as a collineation group is $(1,1)$ isomorphic with this quotient group.

\section{$\S 3$.}

It may be shown that each of these three groups is "transitive" and "primitive" in the variables on which it is represented. By a transitive collineation group is meant one in which the matrix of the coefficients of every conjugate set of linear expressions is not 0 . It follows from the work of Maschke* that an intransitive group is completely reducible, i. e., that the variables may be separated into sets such that each variable is replaced by a linear combination of those in the same set by all the transformations of the group. If the group is also primitive in the sense in which Blichfeldt uses the term, there exist no systems of intransitivity which are permuted by the group.

The group in the $p^{m}$ variables is readily seen to be transitive. The only linear expressions left invariant except for a multiplier by each of the transformations $A_{i}$ are the $p^{m}$ variables themselves. Since the group generated by the $A$ 's is commutative as a linear group as well as a collineation group, any linear system of the variables containing $k$ homogeneous parameters which is left unaltered must contain $k$ expressions which are left individually unaltered except for a multiplier, i. e., $k$ of the variables themselves. But no such set is left invariant by all the $B$ 's. Hence the invariant subgroup is itself transitive in the variables.

We consider now the group commutative with $R$. If two variables with subscripts $\xi_{1} \xi_{2} \cdots \xi_{m}$ and $\eta_{1} \eta_{2} \cdots \eta_{m}$ have the same multiplier under each of the transformations $L_{i}, N_{i j}$, we must have $\xi_{i}{ }^{2}=\eta_{i}{ }^{2}, \xi_{i} \xi_{j}=\eta_{i} \eta_{j}(i, j=1,2, \cdots, m)$. Hence $\xi_{i}= \pm \eta_{i}$, where the sign is the same for all values of $i$.

* Math. Annalen, vol. 52 (1899), p. 363. 
Hence of the expressions

$$
X_{0 \cdots 0}, \quad X_{\xi_{1} \cdots \xi_{m}}+X_{-\xi_{1} \cdots-\xi_{m}}, \quad X_{\xi_{1} \cdots \xi_{m}}-X_{-\xi_{1} \cdots-\xi_{m}},
$$

no two in either set have the same multiplier under all the transformations $L_{i}, N_{i j}$.

Hence if there is any system of intransitivity in either set under the whole group commutative with $R$, it must be determined by a certain number of these expressions themselves. But under the product $M_{1} M_{2} \ldots M_{m}, X_{0} \ldots 0$ is transformed into the sum of all the $p^{m}$ variables. Hence the group in the $\left(p^{m}+1\right) / 2$ variables is transitive.

Under $M_{1} M_{2} \ldots M_{m}, X_{10} \ldots 0-X_{-10} \ldots 0$ is transformed into a linear expression involving all the variables in which $\xi_{1} \neq 0$. Under $M_{2} \cdots M_{m}, X_{010} \ldots 0-X_{0-10} \ldots 0$ is transformed into a linear expression involving all the variables in which $\xi_{1}=0, \xi_{2} \neq 0$. In general, under $M_{i} \cdots M_{m}$, $X_{0} \ldots \xi_{i} \ldots 0-X_{0} \ldots-\xi_{i} \ldots 0$ is transformed into an expression involving all the variables in which $\xi_{1}=\ldots=\xi_{i-1}$ $=0, \xi_{i} \neq 0$.

But these $m$ sets of variables are transformed among themselves. For under $M_{1}, X_{0} \ldots \xi_{i} \ldots 0-X_{0} \ldots-\xi_{i} \ldots 0$ is transformed into an expression involving some variables in which $\xi_{1} \neq 0$. Hence the group in $\left(p^{m}-1\right) / 2$ variables is transitive.

All three systems of groups are readily seen to be primitive in the variables on which they are represented. For if there exist systems of intransitivity, each $L_{i}$ must permute some of them in cycles of period $p$ if $p$ is odd and 2 or 4 if $p=2$. If $p$ is odd this is impossible, since each $L_{i}$ has only $(p+1) / 2$ different multipliers and hence cannot transform a linear expression in the variables into $p$ linearly independent expressions. For the same reason it cannot be of period 4 on any systems of intransitivity if $p=2$. But if it is of period 2 on them, its square will leave them all invariant. Since this square belongs to the invariant subgroup of order $2^{2 m}$, that subgroup would have to be intransitive, since all its operators of period 2 are conjugate. But we have already seen that that subgroup is transitive.

University of Pennsyltania. 